\title{
Public Good Indices for Games with Several LeVels of Approval
}

\author{
SÉbastien Courtin ${ }^{\dagger}$ and Bertrand Tchantcho ${ }^{\ddagger}$
}

August 21, 2019

\begin{abstract}
This work focuses on $(j, 2)$ games in which there are several levels of approval in the input, i.e games with $n$ players, $j$ ordered qualitative alternatives in the input level and 2 possible ordered quantitative alternatives in the output. When considering $(j, 2)$ games, we extend the Public Good index $(P G I)$, the Null Player Free index (NPFI) and the Shift index $(S I)$ and provide full characterizations of these extensions.
\end{abstract}

KEYWORDS: $(j, 2)$ games, Shift index, Public Good index, Null player Free Index JEL Classification Numbers: C71;D71.

\section{Introduction}

This paper analyses the relationship between the production of an output and the likelihood of an individual to influence the production of this output. This cardinal

${ }^{*}$ This project was written while B. Tchantcho was visiting the University of Caen. The authors would like to thank this University for its hospitality. This work has also benefited from comments and encouragemen by a number of conference and seminar participants. The authors thank also ane anonymous reviewers and one associate Editor for their useful comments. This research was developed within the CoCoRICo-CoDEC research program (ANR-14-CE24-0007-02).

${ }^{\dagger}$ Normandie Univ, Unicaen, CREM, UMR CNRS 6211, France. Email: sebastien.courtin@unicaen.fr;

'Eniversity of Yaounde I, Advanced Teachers’ Training College, Cameroon; and University of Cergy-Pontoise, THEMA, UMR CNRS 8184, France. Email: btchantcho@yahoo.fr. 
measure can be seen as the extent to which an individual is useful in this production. Such a problem can be modeled with simple games in which it is standard to study the influence of an individual, by using indices. The most famous are the ShapleyShubik (Shapley and Shubik [1954]) index, the Banzhaf index (Banzhaf [1965]) and the Deegan-Packel index (Deegan and Packel [1978]) ${ }^{1}$. The Shapley-Shubik index and the Banzahf index both suppose that the benefit of the production of an output is only assigned to "pivotal" individuals. A pivotal individual changes a coalition from a losing to a winning one. The more an individual is "pivotal" in the production of the good, the bigger his influence and the greater the benefits he derives. Note that these two indices only consider the formation of winning coalitions as relevant, i.e. coalitions that ensure the production of the output. The Deegan-Packel index results from assuming that only the minimal winning coalitions are likely to form intentionally. A winning coalition is a minimal winning coalition when the removal of any of its members would prevent the coalition from the production of the good. Only the individuals who are members of these minimal winning coalitions are supposed to be influential. Intuitively, an individual who is not needed to raise the aggregate output above a certain threshold (a surplus individual), has no influence on the production of the output and, furthermore, has no incentive to be a member of that minimal coalition. Deegan and Packel [1978] suppose that all the players in a minimal winning coalition equally share the benefits of the production of the output. All these indices have a common factor: the value of the coalition, once formed, can be interpreted as a private good, which can therefore be split among the coalition members even though it was created collectively.

In Barry [1980] and Holler [1982] it has been argued that those measures are inadequate since the value of the coalition, once formed, could not be seen as a private good but as a public good. According to these authors, coalition formation is not about sharing benefits (private good) but about producing goods which cannot be produced by separate individuals. Holler [1982] introduced the public good index $(P G I)$, which is based on the principles of non-rivalry in consumption, non-excludability of access, and the non free-riding property. The PGI is an alternative measure assigning influence proportionally to the number of minimal winning coalitions an individual belongs to. As it is the case with Deegan-Packel index, it is assumed that the coalitions that are not minimal are irrelevant when it comes to

\footnotetext{
${ }^{1}$ We refer to Andjiga et al. [2003] and Laruelle and Valenciano [2008] for a detailed description of indices.
} 
measuring influence. Therefore, they should not be taken into consideration since they can potentially free ride when coalitions determine the production of public goods.

Following the approach of Holler [1982], Alonso-Meijide and Freixas [2010] and Alonso-Meijide et al. [2011] introduced two extentions of the PGI when considering a bigger or smaller set of winning coalitions. Alonso-Meijide et al. [2011] introduced the Null Player Free index (NPFI) in which they evaluate influence by considering the set of null player free winning coalitions. A winning coalition is said to be a null player free winning coalition if no null player belongs to the coalition. In other words, only a coalition in which there are no "free riders" must be taken into account to evaluate the influence of the individual. Note that such coalitions are less restrictive than minimal ones since there are no "free riders" in the latter, as well as no "surplus individuals". Alonso-Meijide and Freixas [2010] introduced the Shift index (SI) based on the PGI and the Riker's principle (Riker [1982]). They introduced the notion of shift minimal winning coalitions, in which the winning coalition that is finally formed to produce a good, contains no surplus individuals. Moreover, no individual within it can be replaced by a weaker individual because, in this case, the status of winning coalition would no longer be maintained.

It has been pointed out by many scholars that a number of interesting questions in economics, politics and more generally social sciences cannot be described by a simple game. Indeed, the observation of real life shows that several levels of participation in public decisions or collective productions are often present. This goes some way towards explaining the introduction of $(j, 2)$ games by Freixas and Zwicker [2003a]. These are games in which individuals choose from among $j$ levels of approval their degree of contribution to the production of the output. These individual choices partition the whole society into $j$ coalitions, each possible partition facing two levels of collective approval. This class of games generalizes the class of simple games which allow only two levels of approval: to participate in the production and to not participate in the production. The final outcome being "to produce" or "not to produce" depending on whether the final bipartition resulting from the individual choices. ${ }^{2}$

Freixas [2005a] and Freixas [2005b] extend the Shapley-Shubik and the Banzhaf indices to $(j, 2)$ games. However, one can formulate the same criticism as Holler

\footnotetext{
${ }^{2}$ There is a large literature on game in which there are multiple alternatives of support, see Courtin et al. [2016] and Courtin et al. [2017] for a recent review.
} 
[1982] for these two extensions. We argue that the influence of an individual (who is not a free-rider) does not depend on her level of contribution to the production of an output. Whatever their level of contributions, individuals belonging to a partition that ensures the production of the output (a winning partition) must have the same influence. The difference of influence between two individuals depends on the number of winning partitions these individuals belong to. Our main contribution is to extend and fully characterize the PGI, the NPFI and the SI when $(j, 2)$ games are taken into consideration

This work is structured as follows. The next section introduces some notations and presents the considered concept of games. Section 3 defines and characterizes the PGI for $(j, 2)$ games. Section 4 extends our analysis to the NPFI and SI index, while Section 5 concludes the paper.

\section{Preliminaries}

\subsection{Simple game}

A finite set of individuals (players) is denoted by $N=\{1,2, \ldots, n\}$ and $2^{N}$ is the set of all subsets of $N$. Any non-empty subset of $N$ is called a coalition. A classical cooperative $n$-person game in characteristic form is a pair $(N, v)$ where $v: 2^{N} \rightarrow \mathbb{R}$ is such that $v(\varnothing)=0$.

Such a game is called a simple game $(S G)$ if : (i) $v(S) \in\{0,1\}$ for all $S \in 2^{N}$; (ii) $v(N)=1$; and (iii) $v$ is monotonic $(v(S) \leq v(T)$ if $S \subseteq T \subseteq N)$. In a $S G$, a coalition $S$ is said to be winning if $v(S)=1$ and losing otherwise. $W(v)$ denotes the set of winning coalitions of the game $(N, v)$. A coalition $S$ is a minimal winning coalition in $(N, v)$ if $v(S)=1$ and $v(T)=0$ for any $T \subset S . M(v)$ is the set of minimal winning coalitions of the game $(N, v)$, while $M_{p}(v)$ is the subset of minimal winning coalitions formed by coalitions $S \subseteq N$ such that $p \in S$. An individual is a null player if she does not belong to any minimal winning coalition. For the sake of simplicity, a game $(N, v)$ will be denoted $v$ when there is no ambiguity.

\subsection{Index}

An index is a mapping $\varphi$ assigning to each $S G$ a $n$-dimensional real-valued vector $\varphi(v)=\left(\varphi_{1}(v), \ldots, \varphi_{n}(v)\right) . \varphi_{i}(v)$ can be interpreted as the influence (power) of player 
$p \in N$ in the game $v$. In this paper we focus our attention on the indices, in which the set of minimal winning coalitions plays an important role.

\section{Public Good index}

Holler [1982] supposes that only minimal winning coalitions are relevant when it comes to measuring influence. He introduced the Public Good Index (PGI) which assigns to each player $p \in N$ the real number:

$$
h_{p}(v)=\frac{\left|M_{p}(v)\right|}{\sum_{p \in N}\left|M_{p}(v)\right|}
$$

The PGI of a player $p$ is equal to the total number of minimal winning coalitions containing player $p$, normalized by the sum of these numbers for all players.

Holler and Packel [1983] show that the PGI is the unique index that satisfies the axioms of null player, efficiency, symmetry and PGI-mergeability. Following the first axiom, a null player gets nothing. Efficiency states that the sum of the individual influence shares equals one. Symmetry says that if two individuals play the same role, then they should have the same influence. Finally, PGI-mergeability implies that influence in a union game is a weighted mean of the influence of the two component games, where the weights come from the number of minimal winning coalitions in each component game.

An alternative characterization is due to Alonso-Meijide et al. [2008] who replaced the axiom of PGI-mergeability with the property of PGI-minimal monotonicity. According to this axiom, if the set of minimal winning coalitions containing a player $p$ in a game $w$ is a subset of the minimal winning coalitions containing this player in another game $v$, then the influence of player $p$ in the game $v$ is not less than the influence of player $p$ in the game $v$, once this influence is normalized by the number of minimal winning coalitions of every player in games $v$ and $w$.

\section{Null Player Free index}

Alonso-Meijide et al. [2011] extend the PGI when considering a set of coalitions bigger than the set of minimal winning coalitions and smaller than the set of winning coalitions. This set is based on the notion of a null player. A winning coalition $S$ is a null player free winning coalition if there is no null player $p \in S$. The set of null player free winning coalitions will be denoted by $N P F(v)$ and the set of null player 
free winning coalitions which $p$ belongs to by $N P F_{p}(v)$. The Null player Free Index $(N P F I)$ assigns to each player $p \in N$ the real number

$$
n p f_{p}(v)=\frac{\left|N P F_{p}(v)\right|}{\sum_{p \in N}\left|N P F_{p}(v)\right|} .
$$

In order to characterize this index, Álvarez-Mozos [2012] intoduced the null player free-minimal monotonicity (NFP-minimal monotonicity), which follows the same idea of the PGI-minimal monotonicity, when considering null player free winning coalitions. The NPFI index is the only index that satisfies the efficiency, symmetry, null player and NFP-minimal monotonicity axioms.

\section{Shift index}

Alonso-Meijide and Freixas [2010] argue that not all the minimal winning coalitions will be formed, and use a smaller set of minimal winning coalitions named shift winning coalitions. A shift minimal coalition is a minimal winning coalition which is stable, in the sense that no player in it can be replaced by a weaker player, because the new coalition becomes a losing coalition. In order to make the notion of a weaker player clearer we need to recall the desirability relation between players introduced by Isbell [1958].

In a simple game $v, p$ is said to be more desirable than $q$, denoted by $p>q$ if the following two conditions are fulfilled: i) for every coalition $S$ such that $p \notin S$ and $q \notin S, S \cup q \in W(v) \Rightarrow S \cup p \in W(v)$; ii) there exists a coalition $T$ such that $p \notin T$ and $q \notin T, T \cup p \in W(v)$ and $T \cup q \notin W(v)$. Players $p$ and $q$ are said to be equally desirable, denoted by $p \sim q$ if: for any coalition $S$ such that $p \notin S$ and $q \notin S$, $S \cup p \in W(v) \Longleftrightarrow S \cup q \in W(v)$.

The desirability relation (or the influential relation) denoted by $\geq$ is defined on $N$ as follows: $p \geq q$ if $p>q$ or $p \sim q$.

We can now introduce the notion of shift minimal winning coalition. For a game $v$ and its desirability relation $\geq$, a coalition $S \in M(v)$ is shift minimal if for every $p \in S$ and $q \notin S$ such that $p>q$, it holds $(S \backslash p) \cup q \notin W(v)$. The set of shift minimal coalitions will be denoted by $S M(v)$.

Alonso-Meijide and Freixas [2010] assume that only shift minimal winning coalitions are relevant and then introduce the Shift Index $(S I)$ which assigns to each player $p \in N$ the real number:

$$
s_{p}(v)=\frac{\left|S_{p}(v)\right|}{\sum_{p \in N}\left|S_{p}(v)\right|}
$$


where $S_{p}(v)$ is the set of shift minimal coalitions $S$ such that $p \in S$.

Alonso-Meijide and Freixas [2010] showed that this index also satisfies the null player, efficiency, symmetry axioms, as well as the shift-minimal monotonicity. The later axiom is a monotonicity axiom when considering shift minimal winning coalitions instead of minimal winning coalitions.

\section{Example}

To conclude this subsection, let us illustrate the various concepts introduced above through the following example. Let the game $v$ on $N=\{1,2,3,4,5,6\}$ be defined by the following set of minimal winning coalitions $M(v)=\{(12),(13),(14),(15),(234),(235),(245)\}$. It ensues that the desirability relation is $1>2>3 \sim 4 \sim 5>6$. Note that 6 is a null player. The different sets $S M(v), N P F(v)$ and $W(v)$ are presented in the table below in which we can see that $S M(v) \subset M(v) \subset N P F(v) \subset W(v)$.

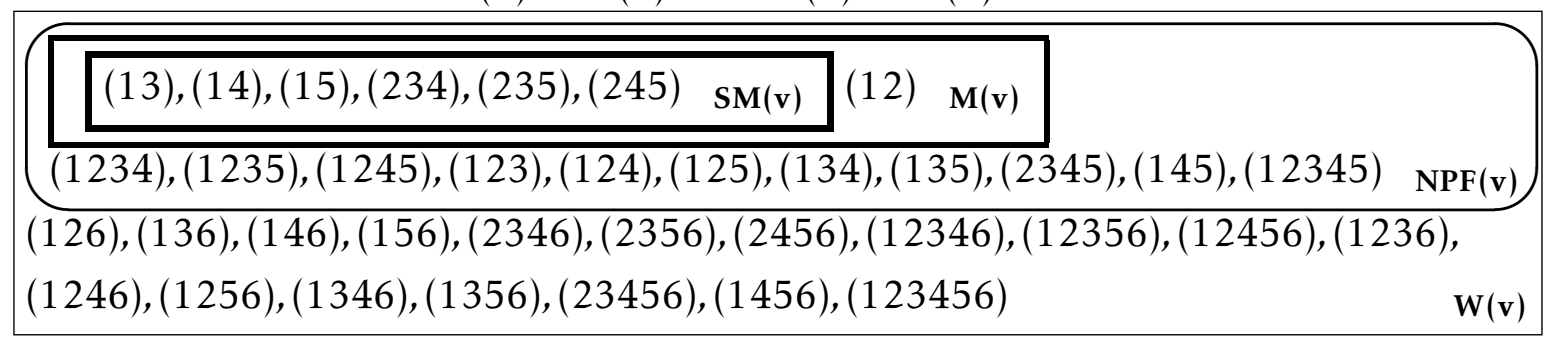

After taking into account the different sets of minimal winning coalitions of each of the individuals, we obtain these indices:

$$
\begin{gathered}
s(v)=\left(\frac{3}{15}, \frac{3}{15}, \frac{3}{15}, \frac{3}{15}, \frac{3}{15}, 0\right) \\
h(v)=\left(\frac{4}{17}, \frac{4}{17}, \frac{3}{17}, \frac{3}{17}, \frac{3}{17}, 0\right) \\
n p f(v)=\left(\frac{14}{56}, \frac{12}{56}, \frac{10}{56}, \frac{10}{56}, \frac{10}{56}, 0\right)
\end{gathered}
$$

We observe that: i) Player 6 who is a null player has always zero power. ii) The Shift index gives the same power to all the players (as the exception of 6). Since the minimal coalition (12) is outside the set of shift minimal coalitions, the power of 1 and 2 decrease whereas those of 4 and 5 increase with the Shift index. iii) The distribution of power between the Public good index and the Null player free index is similar.

\section{$2.3(j, 2)$ Games}

For any positive integer $r$, let $I_{r}$ denote the set $\{1,2, \ldots, r\}$. An ordered $j$-partition of $N$ is a sequence $\mathcal{A}=\left(A_{1}, A_{2}, \ldots, A_{j}\right)$ of mutually disjoint subsets of $N$ whose union 
is $N$. Any subset $A_{l}, l \in I_{j}$ is allowed to be empty, and we think of $A_{l}$ as the set of individuals whose level of approval is $l$. The highest and lowest levels of approval are, respectively, 1 and $j$. We denote by $\mathcal{N}^{j}$ the set of all ordered $j$-partitions of $N$. For any ordered $j$-partition $\mathcal{A}$ and for any $l \in I_{j}$, we define $\uparrow \mathcal{A}_{l}=A_{1} \cup A_{2} \cup \ldots \cup A_{l}$. Let $\mathcal{A}^{1}$ and $\mathcal{A}^{2}$ be two $j$-partitions. We write $\mathcal{A}^{1} \subseteq^{j} \mathcal{A}^{2}$ if $\mathcal{A}^{1}$ can be transformed into $\mathcal{A}^{2}$ by shifting one or more voters to higher levels of approval. That is, $\mathcal{A}^{1} \subseteq^{j} \mathcal{A}^{2} \Leftrightarrow$ $\forall l \in I_{j}, \uparrow \mathcal{A}_{l}^{1} \subseteq \uparrow \mathcal{A}_{l}^{2}$. Finally $\mathcal{A}^{1} \subsetneq^{j} \mathcal{A}^{2}$ means: $\mathcal{A}^{1} \subseteq^{j} \mathcal{A}^{2}$ and $\mathcal{A}^{1} \neq \mathcal{A}^{2}$.

A $(j, 2)$ game $(N, V)$, with $j \geq 2$, consists of a finite set $N$ of players together with a value function $V: \mathcal{N}^{j} \rightarrow\left\{v_{1}, v_{2}\right\}$ where $\left\{v_{1}, v_{2}\right\}$ is the value set of $(N, V)$ whose elements are any 2 objects equipped with a strict linear ordering $v_{1}>v_{2}$ and such that for all ordered $j$-partitions $\mathcal{A}^{1}$ and $\mathcal{A}^{2}$, if $\mathcal{A}^{1} \subseteq j \mathcal{A}^{2}$, then $V\left(\mathcal{A}^{2}\right)=V\left(\mathcal{A}^{1}\right)$, or $V\left(\mathcal{A}^{2}\right)>V\left(\mathcal{A}^{1}\right)$. Note that in a $(j, 2)$ game, $V=(N, \emptyset, \ldots, \emptyset)=v_{1}$ and $V=(\emptyset, \emptyset, \ldots, N)=$ $v_{2}$.

In the following, we let $v_{1}=$ win and $v_{2}=$ lose, and we denote by $\mathcal{W}(V)$ the set of all $j$-partitions $\mathcal{A}$ of $N$ such that $V(\mathcal{A})=$ win. Those $j$-partitions will be referred to as winning $j$-partitions. ${ }^{3}$ From the set of winning $j$-partitions, we can define the set of minimal winning $j$-partitions, $\mathcal{M}(V)$. A $j$-partition $\mathcal{A}^{1}$ is a minimal winning $j$-partition whenever $\mathcal{A}^{1}$ is a winning $j$-partition and $\mathcal{A}^{2}$ is a losing $j$-partition if $\mathcal{A}^{2} \subseteq^{j} \mathcal{A}^{1}$. This means that if an output can be produced with a smaller contribution of at least one of the individuals, the $j$-partition is not a minimal one.

The purpose of $(j, 2)$ games is to generalize simple games where only "yes" and "no" votes are considered $(j=2)$. In the following, when there can be no confusion, a $(j, 2)$ game $(N, V)$ will be denoted $V$.

Note that for any $j$-partition $\mathcal{A}$ and any player $p, \mathcal{A}(p)$ denotes the level of approval of $p$ in $\mathcal{A}$ and $\mathcal{A}_{p}^{r}$, the $j$-partition in which player $p$ (alone) moves, from $\mathcal{A}(p)$ to level $r$.

In order to illustrate the terminology of a $(j, 2)$ game, Freixas and Zwicker [2003b] present the following real situation. At the United Nation Security Council, a resolution is carried if at least nine members support it and no permanent member is explicitly opposed. Let $P=\{1,2,3,4,5\}$ and $R=\{6,7, \ldots, 15\}$ be the set of permanent members and non-permanent members respectively.The corresponding $(j, 2)$ game is given by:

\footnotetext{
${ }^{3}$ For a numerical representation of a $(j, 2)$ game, we set, as usual, $V(\mathcal{A})=1$ for any winning $j$ partition and $V(\mathcal{A})=0$ for any losing one.
} 


$$
V(\mathcal{A})=V\left(A_{1}, A_{2}, A_{3}\right)=\left\{\begin{array}{l}
1 \text { if }\left|A_{1}\right| \geq 9 \text { and } A_{3} \cap P=\varnothing \\
0 \text { otherwise }
\end{array}\right.
$$

with $A_{1}$ being the set of voters of $\mathrm{N}$ who vote "yes"; $A_{2}$ the set of voters of $\mathrm{N}$ who "abstain"; and $A_{3}$ the set of voters of $\mathrm{N}$ who vote "no".

\section{The Public Good index for $(j, 2)$ games}

In this section, we extend the PGI to $(j, 2)$ games, and we outline two axiomatic approaches for this new index in this context.

Considering $(j, 2)$ games, an index is a mapping $\phi$ which assigns to each $(j, 2)$ game a vector $\phi(V)=\left(\phi_{1}(V), \ldots, \phi_{n}(V)\right)$. Let $\mathcal{C}_{p}(V)=\left\{\mathcal{A} \in \mathcal{M}(V): p \notin A_{j}\right\}$ and $c_{p}(V)=$ $\left|\mathcal{C}_{p}(V)\right| . c_{p}(V)$ expresses the number of minimal $j$-partitions $\mathcal{A}$ in which player $p$ does not chose the last level of approval $j$. The PGI for $(j, 2)$ games is then given by

$$
H_{p}(V)=\frac{c_{p}(V)}{c(V)} .
$$

where $c(V)=\sum_{p \in N} c_{p}(V)$.

In a $(j, 2)$ game, the $P G I$ of a player $p$ is equal to the total number of minimal winning $j$-partitions in which player $p$ does not chose the last level of approval, normalized by the sum of these numbers for all players.

The PGI for $(j, 2)$ games presented above is based on three principles:

1. Along the spirit of Holler [1982], we choose to not give an amount of power to the surplus players and to the free riders players. It is assumed that some winning partitions are not stable and they should not be taken into consideration for measuring the power. Indeed, in some winning partitions a player might feel unhappy because she think that she could obtain more power by excluding some players. Hence, our first principle consists in consider only minimal winning $j$-partition, in which there is neither surplus player nor free rider player.

2. To complete our analysis, one needs to add a second principle. Contrary to simple game, in which all minimal winning coalitions are taking into account to compute the index, this extension excludes some minimal $j$-partitions. Since all the players belongs to a minimal $j$-partition, not excluding $j$-partitions would lead to give an equal power to all the players whatever the game. We 
choose to not give power to players who contribute at the last level of support. As an example, in $(2,2)$ games in which the players can participate or not to the production of the good, this implies that we do not give power to a player who chooses to not participate.

3. Finally, we assume that all the other players must be rewarded in the same way, whatever their contributions along the original idea behind the PGI index. Once a public good is delivered, all the players must benefit of it, whatever their own contribution and the way in which others contribute (as the exception of those who do not contribute). In other words, players can have different contributions, but sharing the same amount of power.

Let us now introduce some axioms.

A player $p$ is called a null player if $p \in A_{j}$ for every minimal winning $j$-partition $\mathcal{A}$. A null player can never help an arbitrary losing $j$-partition to win.

Let $\phi$ be an index for $(j, 2)$ games.

Axiom 1. (Null player)

$p$ is a null player for $V$ implies $\phi_{p}(V)=0$

Axiom 2. (Efficiency)

$\sum_{p=1}^{n} \phi_{p}(V)=1$

Before to present the next two axioms, one need to introduce the following notation. For a given minimal $j$-partition $\mathcal{A}$, let $I_{p}(\mathcal{A})$ the fraction of power receives by player $p$.

Axiom 3. (Minimal Additivity)

$\phi$ satisfies minimal additivity for $(j, 2)$-games if for any $V$, for all player $p$,

$$
\phi_{p}(V)=\sum_{\mathcal{A} \in \mathcal{M}(V)} I_{p}(\mathcal{A})
$$

Axiom 4. (Minimal Homogeneity)

$\phi$ satisfies minimal homogeneity for $(j, 2)$-games if for any $\mathcal{A} \in \mathcal{M}(V)$ and for all $p, q \notin A_{j}$,

$$
I_{p}(\mathcal{A})=I_{q}(\mathcal{A})
$$

According to Axiom 1, a player who always contributes to the last level in a minimal winning $j$-partition has no influence. Axiom 2 means that the sum of the influence is equal to one. Axiom 3 implies that the power of a player $p$ depends on 
the fraction of power received in each minimal $j$-partition considered to evaluate the power. Axiom 4 states that the amount of power of a player in each minimal $j$-partition is the same whatever her relative position inside the partition.

In most of influence (power) indices characterization, the key axiom is usually an additive axiom, which relates the influence in two separate games $(N, V)$ and $(N, W)$ to the influence in a certain join game derived from of $(N, V)$ and $(N, W)$. For $(j, 2)$ games in general (and for simple games in particular), the Shapley-Shubik influence index verifies the transfer axiom, which is not the case of the PGI index. However it satisfies a "similar" property, the merger axiom.

Let $(N, V)$ and $(N, W)$ be two $(j, 2)$ games. The $(j, 2)$ game $(N, V \vee W)$ is defined as follow. For any j-partition $\mathcal{A}, V \vee W(\mathcal{A})=\max (V(\mathcal{A}), W(\mathcal{A})$ ). The games $V$ and $W$ are said to be mergeable if for all $\mathcal{A} \in \mathcal{M}(V)$ it holds $\mathcal{A} \notin \mathcal{W}(W)$ and for all $\mathcal{A} \in \mathcal{M}(W)$ it holds $\mathcal{A} \notin \mathcal{W}(V)$.

If two games $(N, V)$ and $(N, W)$ are mergeable, the minimal winning $j$-partitions in the max game $(N, V \vee W)$ are precisely the union of the minimal winning $j$ partitions in the two separate games $(N, V)$ and $(N, W)$. In other words, the minimal $j$-partitions related to the two separate games are also minimal $j$-partitions for the $\max$ game. It is easy to see that this condition guarantees that $|\mathcal{M}(V \vee W)|=|\mathcal{M}(V)|$ $+|\mathcal{M}(W)|$.

\section{Axiom 5. (Mergeability)}

$V$ and $W$ mergeable implies that for all $p \in N$,

$$
\phi_{p}(V \vee W)=\frac{c(V)}{c(V \vee W)} \phi_{p}(V)+\frac{c(W)}{c(V \vee W)} \phi_{p}(W)
$$

According to the mergeability axiom, the influence in a merged game is a weighted mean of the influence of the two component games. The weights come from the number of minimal winning $j$-partitions (in which the player $p$ does not choose the last level of support) in each component game, divided by the number of minimal winning $j$-partitions (in which the player $p$ does not choose the last level of support) in the merged game. Hence, it is similar to the transfer axiom in the sense that it assesses the influence in a merged game in terms of the influence in the two component games.

Note that when considering influence of an individual in a $(j, 2)$ game (as in simple game), we are not dealing with strategic consideration of that individual. Indeed, the measure of influence take into account the information embodied in the 
decision-making process modelled in the game considered. Of course, the individual characteristics and preference influence the final outcome. But such information is left aside when considering influence index. The influence indices are intended to quantify the a priory implication of a decision-making rule, not any particular application of them.

Back to the merger axiom, it is true that the behavior of an individual in the games $(N, V)$ and $(N, W)$ can be quite different to the behavior in the game $(N, V \vee$ $W)$, however one can not take into account this information when we measure the influence. ${ }^{4}$.

Theorem 1. Let $\phi$ be an index for $(j, 2)$ games. Then $\phi$ satisfies Axiom 1, Axiom 2, Axiom 3, Axiom 4 and Axiom 5 if and only if $\phi=H$.

Proof. ${ }^{5}$

\section{Existence}

It is obvious that $H$ satisfies the efficiency, the null player and the minimal homogeneity axioms. The minimal additivity axiom is verified since for all $p \in N$, we have $H_{p}(V)=\sum_{\mathcal{A} \in \mathcal{M}(V)} I_{p}(\mathcal{A})$ with $I_{p}(\mathcal{A})=\left\{\begin{array}{l}\frac{1}{c(V)} \text { if } p \notin A_{j} \\ 0 \text { otherwise }\end{array}\right.$

Let us prove that $H$ satisfies the mergeability axiom. Let $V_{1}$ and $V_{2}$ be two $(j, 2)$ games on $N$ and $p \in N$. If $V_{1}$ and $V_{2}$ are mergeable then, it is easy to verify that $\mathcal{C}_{p}\left(V_{1} \vee V_{2}\right)=\mathcal{C}_{p}\left(V_{1}\right) \cup \mathcal{C}_{p}\left(V_{2}\right)$ and $\mathcal{C}_{p}\left(V_{1}\right) \cap \mathcal{C}_{p}\left(V_{2}\right)=\emptyset$, which implies that $c_{p}\left(V_{1} \vee V_{2}\right)=$ $c_{p}\left(V_{1}\right)+c_{p}\left(V_{2}\right)$. Therefore,

$$
\begin{aligned}
H_{p}\left(V_{1} \vee V_{2}\right)=\frac{c_{p}\left(V_{1} \vee V_{2}\right)}{c\left(V_{1} \vee V_{2}\right)} & =\frac{c_{p}\left(V_{1}\right)+c_{p}\left(V_{2}\right)}{c\left(V_{1} \vee V_{2}\right)} \\
& =\frac{c_{p}\left(V_{1}\right)}{c\left(V_{1} \vee V_{2}\right)}+\frac{c_{p}\left(V_{2}\right)}{c\left(V_{1} \vee V_{2}\right)} \\
& =\frac{H_{p}\left(V_{1}\right) \cdot c\left(V_{1}\right)}{c\left(V_{1} \vee V_{2}\right)}+\frac{H_{p}\left(V_{2}\right) \cdot c\left(V_{2}\right)}{c\left(V_{1} \vee V_{2}\right)} \\
& =\frac{c\left(V_{1}\right)}{c\left(V_{1} \vee V_{2}\right)} H_{p}\left(V_{1}\right)+\frac{c\left(V_{2}\right)}{c\left(V_{1} \vee V_{2}\right)} H_{p}\left(V_{2}\right)
\end{aligned}
$$

\section{Uniqueness}

Conversely, let $\phi$ be an index which satisfies efficiency, null player, minimal additivity and minimal homogeneity axioms. We shall prove that $\phi=H$. In order to

\footnotetext{
${ }^{4}$ We thanks a Reviewer for this remark

${ }^{5}$ The arguments used in the proof are very similar to those used in Holler and Packel [1983] to characterize the Public Good Index for simple game. The main difference resides in the fact that here we consider $(j, 2)$ games.
} 
do so, let $V$ be a $(j, 2)$ game and $p \in N$. We will proceed by induction on the number of minimal winning $j$-partitions of $V$. Assume that $M(V)=\left\{\mathcal{A}=\left(A_{1}, A_{2}, \ldots, A_{j}\right)\right\}$ that is, $|M(V)|=1$. Since $|M(V)|=1$, then by minimal additivity $\phi_{p}(V)=I_{p}(\mathcal{A})$. From minimal homogeneity $I_{p}(\mathcal{A})=I_{q}(\mathcal{A})$ for all $p, q \notin A_{j}$. From efficiency, it follows that $\sum_{q \in N} I_{q}(\mathcal{A})=1$. Moreover $\sum_{q \in N} I_{q}(\mathcal{A})=\sum_{q \in A_{j}} I_{q}(\mathcal{A})+\sum_{q \notin A_{j}} I_{q}(\mathcal{A})=\sum_{q \notin A_{j}} I_{q}(\mathcal{A})$ because from the Null player axiom, $I_{q}(\mathcal{A})=\phi_{q}(V)=0$ for all $q \in A_{j}$. But, for all $q \notin A_{j}$, $\sum_{q \notin A_{j}} I_{q}(\mathcal{A})=\sum_{q \notin A_{j}} I_{p}(\mathcal{A})=I_{p}(\mathcal{A}) .\left|N \backslash A_{j}\right| ;$ thus, $I_{p}(\mathcal{A})=\frac{1}{\left|N \backslash A_{j}\right|}=\phi_{p}(V)$ for all $p \notin A_{j}$. Note that $N \backslash A_{j} \neq \emptyset$ because from the definition of a $(j, 2)$ game, $V(\emptyset, \ldots, \emptyset, N)=0$. On the other hand, we know that $H_{p}(V)=\left\{\begin{array}{l}\frac{1}{\left|N \backslash A_{j}\right|} \text { if } p \notin A_{j} \\ 0 \text { otherwise }\end{array}\right.$. Thus, $H_{p}(V)=\phi_{p}(V)$.

Let $r>1$ and assume that $H_{p}(V)=\phi_{p}(V)$ holds for all game $V$ for which there are at most $r-1$ minimal winning $j$-partitions. Consider a game $V$ with $r$ minimal winning $j$-partitions, say $\mathcal{A}^{1}, \mathcal{A}^{2}, \ldots, \mathcal{A}^{r}$.

The games $V_{1} \vee \ldots \vee V_{r-1}$ and $V_{r}$ are mergeable, where $V_{r}$ is the $(j, 2)$ game with the single minimal $j$-partition $\mathcal{A}^{r}$.

Furthermore, $V=\left[V_{1} \vee \ldots \vee V_{r-1}\right] \vee V_{r}$ and it follows, from the mergeability axiom that

$$
\begin{aligned}
\phi_{p}(V)= & \frac{c_{p}\left(V_{1} \vee \ldots \vee V_{r-1}\right)}{c(V)} \phi_{p}\left(V_{1} \vee \ldots \vee V_{r-1}\right)+\frac{c_{p}\left(V_{r}\right)}{c(V)} \phi_{p}\left(V_{r}\right) \\
= & \frac{c_{p}\left(V_{1} \vee \ldots \vee V_{r-1}\right)}{c(V)} H_{p}\left(V_{1} \vee \ldots \vee V_{r-1}\right)+\frac{c_{p}\left(V_{r}\right)}{c(V)} H_{p}\left(V_{r}\right) \\
& (\text { thanks to the induction hypothesis) } \\
= & \frac{1}{c(V)} c_{p}\left(\left[V_{1} \vee \ldots \vee V_{r-1}\right] \vee V_{r}\right) \text { (because } H \text { satisfies the mergeability axiom) } \\
= & \left.\frac{c_{p}(V)}{c(V)} \text { (because of the mergeability of } V_{1} \vee \ldots \vee V_{r-1} \text { and } V_{r}\right) \\
= & H_{p}(V)
\end{aligned}
$$

In conclusion, for all game $V$ on $N$, all player $p \in N, \phi_{p}(V)=H_{p}(V)$.

We now put forward a second axiomatic characterization based on the property of PGI minimal monotonicity. Following the PGI minimal monotonicity for simple games, this property compare the behavior of a player $p$ in two different games $V$ and $W$ in which the players are the same and in which the two sets of minimal winning $j$-partitions are linked. 
Axiom 6. (PGI-minimal monotonicity)

$\phi$ satisfies PGI-minimal monotonicity for $(j, 2)$ games if for any pair $(N, V)$ and $(N, W)$,

$$
\phi_{p}(V) c(V) \geq \phi_{p}(W) c(W)
$$

for all player $p \in N$ such that $\mathcal{C}_{p}(W) \subseteq \mathcal{C}_{p}(V)$

According to this axiom if the set of minimal winning $j$-partitions containing a player $p$ in game $W$ is a subset of the set of minimal winning $j$-partitions containing the same player $p$ in another game $V$, then the influence of player $p$ in game $V$ is not less than power of player $p$ in game $W$. In other words, if player $p$ improves her position in a game, her influence in the game must increase.

Theorem 2. Let $\phi$ be an index for $(j, 2)$ games. Then $\phi$ satisfies Axiom 1, Axiom 2, Axiom 3, Axiom 4 and Axiom 6, if and only if $\phi=H$.

\section{Proof. Existence}

We know that $H$ satisfies the efficiency, null player, minimal additivity and minimal homogeneity axioms. In addition $H$ satisfies the minimal monotonicity axiom because if $p$ is a player such that $\mathcal{C}_{p}\left(V_{1}\right) \subseteq \mathcal{C}_{p}\left(V_{2}\right)$, then $c_{p}\left(V_{1}\right) \leq c_{p}\left(V_{2}\right)$ which means that $H_{p}\left(V_{1}\right) c\left(V_{1}\right) \leq H_{p}\left(V_{2}\right) c\left(V_{2}\right)$.

\section{Uniqueness}

Conversely, let $\phi$ be an index which satisfies efficiency, null player, minimal additivity, minimal homogeneity and minimal monotonicity. Let $V$ be a $(j, 2)$ game and $p \in N$. Again, we will proceed by induction on the number of minimal winning $j$-partitions of $V$.

Once more if $|M(V)|=1$, as we have done before, we prove that $\phi_{p}(V)=H_{p}(V)$.

Now assume that $H_{p}(V)=\phi_{p}(V)$ holds for all game $V$ for which there are at most $r-1$ minimal winning $j$-partitions and consider a game $V$ with $r>1$ minimal winning $j$-partitions, say $\mathcal{A}^{1}, \mathcal{A}^{2}, \ldots, \mathcal{A}^{r}$.

Now, set $T=\bigcap_{t=1}^{r}\left(N \backslash A_{j}^{t}\right)$ and let $p \in N$ :

First case: assume that $p \notin T$. Consider the $(j, 2)$ game $V_{0}$ defined such that $M\left(V_{0}\right)=\left\{\mathcal{A}^{t} \in M(V): p \in N \backslash A_{j}^{t}\right\}$.

By definition of $V_{0}$, it follows $\mathcal{C}_{p}\left(V_{0}\right)=\mathcal{C}_{p}(V)$, which, by the axiom of minimal monotonicity yields $\phi_{p}\left(V_{0}\right) c\left(V_{0}\right) \leq \phi_{p}(V) c(V) \leq \phi_{p}\left(V_{0}\right) c\left(V_{0}\right)$, that is, $\phi_{p}\left(V_{0}\right) c\left(V_{0}\right)=$ $\phi_{p}(V) c(V)$. 
Since $p \notin T$, the $(j, 2)$ game $V_{0}$ has less than $r$ minimal winning $j$-partitions. Thus, $\phi_{p}\left(V_{0}\right)=H_{p}\left(V_{0}\right)$ thanks to the induction hypothesis. On the other hand, as $H$ satisfies the minimal monotonicity axiom, we have $H_{p}\left(V_{0}\right) c\left(V_{0}\right)=H_{p}(V) c(V)$.

We conclude that $\phi_{p}(V) c(V)=\phi_{p}\left(V_{0}\right) c\left(V_{0}\right)=H_{p}\left(V_{0}\right) c\left(V_{0}\right)=H_{p}(V) c(V)$, which implies $\phi_{p}(V)=H_{p}(V)$.

Second case: $p \in T$

By the minimal homogeneity axiom, we have $\phi_{p}(V)=\phi_{q}(V)$ and $H_{p}(V)=H_{q}(V)$ for all $p, q \in T$, and furthermore for all $u \notin T, \phi_{u}(V)=H_{u}(V)$

By efficiency (of $\phi$ and $H$ ), we have :

$\sum_{u \in T} \phi_{u}(V)+\sum_{u \notin T} \phi_{u}(V)=1=\sum_{u \in T} H_{u}(V)+\sum_{u \notin T} H_{u}(V)$.

This implies that $\sum_{u \in T} \phi_{u}(V)=\sum_{u \in T} H_{u}(V)$, that is, $|T| \phi_{p}(V)=|T| H_{p}(V)$ and finally, $\phi_{p}(V)=H_{p}(V)$.

Let us now evaluate the influence of each member of the United Nation Security Council. One need to first consider the minimal winning $j$-partitions of such game. Such partitions can be split in six types. Table 1 summarizes the 6 types, the number of each types, and gives also the number of times a permanent (non permanent member) is not at the last level of support in each type of partition .

Table 1: United Nation Security Council

\begin{tabular}{|c|c|c|c|}
\hline Type & Total Number & Permanent & Non-Permanent \\
\hline (4 R + 5 P , $\varnothing$, Other $)$ & $\left(\begin{array}{l}9 \\
4\end{array}\right)=126$ & 126 & $\left(\begin{array}{l}8 \\
3\end{array}\right)=56$ \\
\hline$(5 \mathrm{R}+4 \mathrm{P}, 1 \mathrm{P}$, Other $)$ & $\left(\begin{array}{l}9 \\
5\end{array}\right) *\left(\begin{array}{l}5 \\
4\end{array}\right)=630$ & 630 & $\left(\begin{array}{l}8 \\
4\end{array}\right)=70$ \\
\hline$(6 \mathrm{R}+3 \mathrm{P}, 2 \mathrm{P}$, Other $)$ & $\left(\begin{array}{l}9 \\
6\end{array}\right) *\left(\begin{array}{l}5 \\
3\end{array}\right)=840$ & 840 & $\left(\begin{array}{l}8 \\
5\end{array}\right)=56$ \\
\hline (7 R $+2 \mathrm{P}, 3 \mathrm{P}$, Other $)$ & $\left(\begin{array}{l}9 \\
7\end{array}\right) *\left(\begin{array}{l}5 \\
2\end{array}\right)=360$ & 360 & $\left(\begin{array}{l}8 \\
6\end{array}\right)=28$ \\
\hline (8 R+1 P, $4 \mathrm{P}$, Other $)$ & $\left(\begin{array}{l}9 \\
8\end{array}\right) *\left(\begin{array}{l}5 \\
1\end{array}\right)=45$ & 45 & $\left(\begin{array}{l}8 \\
7\end{array}\right)=8$ \\
\hline (9 R, $5 \mathrm{P}$, Other) & 1 & 1 & $\left(\begin{array}{l}8 \\
3\end{array}\right)=1$ \\
\hline Total & 2002 & 2002 & 219 \\
\hline
\end{tabular}

This table shows that the public good index of a permanent member is equal to $\frac{2002}{11981} \simeq 0.1670$ and the public good index of a non-permanent member is equal to $\frac{219}{11981}=0.0183$. Since a permanent member is a veto player, she belongs to all the minimal winning $j$-partitions and she has a higher power . 


\section{Two public good index extensions}

In this section, following the approach of Alonso-Meijide et al. [2011] and AlonsoMeijide and Freixas [2010], we consider two extensions of the PGI. First, we assume a bigger set of minimal $j$-partitions and we introduce the Null Player Free index for $(j, 2)$ games. Second, we restrict the domain of minimal $j$-partitions to shift minimal $j$-partitions, and propose a Shift index for $(j, 2)$ games.

\subsection{The Null Player Free index for $(j, 2)$ games}

Let $\mathcal{N} \mathcal{P} \mathcal{F}(V)=\left\{\mathcal{A} \in \mathcal{W}(V): p\right.$ null player implies $\left.p \in A_{j}\right\}$. This is the set of winning $j$ partitions for which all null players lie at the last level of approval $j$. In the following, we denote by $\mathcal{N P \mathcal { F }}_{p}(V)=\left\{\mathcal{A} \in \mathcal{N} \mathcal{P} \mathcal{F}(V): p \notin A_{j}\right\}$ the set of null player free winning $j$-partitions in which player $p$ is not at the last level. The Null Player Free Index for $(j, 2)$ assigns to each player $p \in N$ the real number

$$
N F_{p}(V)=\frac{\left|\mathcal{N P F}_{p}(V)\right|}{\sum_{p \in N}\left|\mathcal{N P \mathcal { F }}_{p}(V)\right|} .
$$

The difference between NPF index for $(j, 2)$ games and the PGI for $(j, 2)$ games lies in the considered winning $j$-partition. For the $P G I$, these are the minimal $j$-partitions whereas for the NPF index, these are the null player free ones. In other words, when using the NPF index to measure the influence of a player, one needs to consider only winning $j$-partitions in which the "free-rider" players stay at the last level of support. A "free-rider" player is a player who can never help an arbitrary losing $j$ partitions win nor can she prevent a winning one from losing. This extension follows the definition of the null player free winning coalitions in which only winning coalitions whitout null players are considered. A simple game can be represented by a $(2,2)$ game with $\mathcal{A}=\left(A_{1}, A_{2}\right)$. Our definition implies that all the winning $j$-partition with null players in $A_{1}$ are not considered. Note that, in a minimal $j$-partition there are no "free-rider" players but this is not a sufficient condition. One needs to add the absence of surplus contribution to completely describe such partitions.

To characterize this new index, one need to extend the additivity and the homogenity axioms.

Axiom 7. (NPF-minimal Additivity)

$\phi$ satisfies NPF-minimal additivity for $(j, 2)$-games if for any $V$, for all player $p$,

$$
\phi_{p}(V)=\sum_{\mathcal{A} \in \mathcal{N} \mathcal{P} \mathcal{F}(V)} \bar{I}_{p}(\mathcal{A})
$$


$\bar{I}_{p}(\mathcal{A})$ the fraction of power the player $p$ receives when the null player free minimal $j$-partition $\mathcal{A}$ emerges.

Axiom 8. (NPF-minimal Homogeneity)

$\phi$ satisfies NPF-minimal homogeneity for $(j, 2)$ - games if for any $\mathcal{A} \in \operatorname{NPF}(V)$ and for all $p, q \notin A_{j}$,

$$
\bar{I}_{p}(\mathcal{A})=\bar{I}_{q}(\mathcal{A})
$$

Following Álvarez-Mozos [2012], we intoduce the null player free-minimal monotonicity for $(j, 2)$ game.

Axiom 9. (NPF-minimal monotonicity)

$\phi$ satisfies NPF-minimal monotonicity for $(j, 2)$ games if for any pair $(N, V)$ and $(N, W)$,

$$
\phi_{p}(V) \sum_{p \in N}\left|\mathcal{N P \mathcal { F }}_{p}(V)\right| \geq \phi_{p}(W) \sum_{p \in N}\left|\mathcal{N} \mathcal{P} \mathcal{F}_{p}(W)\right|
$$

for all player $p \in N$ such that $\mathcal{N} \mathcal{P} \mathcal{F}_{p}(W) \subseteq \mathcal{N} \mathcal{P} \mathcal{F}_{p}(V)$

Once again, the monotonicity axiom describe the behavior of a player $p$ in two simple games $V$ and $W$ with the same set of players, and in which the set of the null player free winning $j$-partitions in which player $p$ is not at the last level in game $W$ is included in those of game $V$. The axiom states that the influence of player $p$ must be "bigger" in game $V$ than in game $W$ (after normalization).

Theorem 3. Let $\phi$ be an index for $(j, 2)$ games. Then $\phi$ satisfies Axiom 1, Axiom 2, Axiom 7, Axiom 8 and Axiom 9, if and only if $\phi=N P F$.

Proof. The proof follows immediately from a similar reasoning to the one used in the proof of Theorem $2 .^{6}$

\subsection{The shift index for $(j, 2)$ games}

We follow two principles for this second public good extension. Firstly, we assume that every player wishes to form part of a minimal winning $j$ partition. Secondly,

\footnotetext{
${ }^{6}$ The NPF-minimal additivity of the null player free index is given by $\bar{I}_{p}(\mathcal{A})=$ $\left\{\begin{array}{l}\frac{1}{\sum_{p \in N}\left|\mathcal{N} \mathcal{P} \mathcal{F}_{p}(V)\right|} \text { if } p \in N \backslash A_{j} \\ 0 \text { otherwise }\end{array}\right.$
} 
every player prefers to be partner with weaker players, as far as the $j$-partition is winning. Therefore we consider a smaller set than minimal $j$-partitions: the shift minimal $j$-partitions. To introduce these special $j$-partitions one needs to recall the notion of desirability for $(j, k)$ games introduced by Pongou et al. [2011] and Pongou et al. [2017].

\section{Desirability relation for $(j, 2)$ games}

The desirability relation for $(j, 2)$ games, $p \geqslant q$ is defined as follows: for all $\mathcal{A} \in \mathcal{N}^{j}$ such that $\mathcal{A}(p)=\mathcal{A}(q)=l \in\{2, \ldots, j\}$, for all $r<l$, if $\mathcal{A}_{q}^{r} \in \mathcal{W}(V)$ then $\mathcal{A}_{p}^{r} \in \mathcal{W}(V)$.

We say that $p$ is more desirable than $q(p>q)$ if $p \geqslant q$ and not $(q \geqslant p)$. Moreover, $p$ and $q$ are said to be equally desirable, denoted $p \sim q$ if $: p \geqslant q$ and $q \geqslant p .{ }^{7}$

\section{Shift index for $(j, 2)$ games}

Let $V$ be a $(j, 2)$ game and $\geqslant$ be its desirability relation. A $j$-partition $\mathcal{A} \in \mathcal{M}(V)$ is said to be shift minimal if for every $p, q \in N$ such that $\mathcal{A}(p)>\mathcal{A}(q)$ and $p>q$, it holds $\pi_{p q} \mathcal{A} \notin \mathcal{W}(V)$, where $\pi_{p q}$ is the transposition of $p$ and $q$. We denote by $\mathcal{S M}(V)$ the set of shift minimal winning $j$-partitions of $V$. In the following, we use the notation $\mathcal{S M}_{p}(V)=\left\{\mathcal{A} \in \mathcal{S} \mathcal{M}(V): p \notin A_{j}\right\}$ as the set of shift minimal winning $j$-partitions of $V$ in which player $p$ is not at the last level. Its cardinality is given by $s m_{p}(V)=\left|\mathcal{S} \mathcal{M}_{p}(V)\right|$.

The Shift index for $(j, 2)$ game of a player $p$, is defined by :

$$
S_{p}(V)=\frac{s m_{p}(V)}{s(V)} \text { where } s(V)=\sum_{p \in N} s m_{p}(V) \text {. }
$$

In a $(j, 2)$ game, the influence of a player measured by the $S I$ index, consists on counting the number of stable minimal $j$-partitions in which this player belongs to and in which she does not choose the last level of support. In such a context, a stable minimal winning partition is a partition in which it cannot be possible to exchange the level of support of a player $p$ with the level of support of a weaker player $q$ (who has a smaller level of support), without altering the status of the $j$-partition. This means that the $j$-partition obtained from such a replacement does not lead to the production of the good.

\footnotetext{
${ }^{7}$ Properties of the desirability relations can be found in Pongou et al. [2014].
} 
Axiom 10. (Shift-minimal Additivity)

$\phi$ satisfies shift-minimal additivity for $(j, 2)$-games if for any $V$, for all player $p$,

$$
\phi_{p}(V)=\sum_{\mathcal{A} \in \mathcal{S} \mathcal{M}(V)} \hat{I}_{p}(\mathcal{A})
$$

$\hat{I}_{p}(\mathcal{A})$ can be seen as the fraction of power the player $p$ receives when a shift minimal $j$-partition $\mathcal{A}$ emerges.

Axiom 11. (Shift-minimal Homogeneity)

$\phi$ satisfies shift-minimal homogeneity for $(j, 2)$ - games if for any $\mathcal{A} \in \mathcal{S M}(V)$ and for all $p, q \notin A_{j}$,

$$
\hat{I}_{p}(\mathcal{A})=\hat{I}_{q}(\mathcal{A})
$$

Axiom 12. (Shift-minimal monotonicity)

$\phi$ satisfies shift-minimal monotonicity for $(j, 2)$ games if for any pair $(N, V)$ and $(N, W)$,

$$
\phi_{p}(V) s(V) \geq \phi_{p}(W) s(W)
$$

for all player $p \in N$ such that $\mathcal{S M}_{p}(W) \subseteq \mathcal{S M}_{p}(V)$.

The meaning of the three axioms are very similar to the previous one. The difference lies on the minimal $j$-partitions considered.

Theorem 4. Let $\phi$ be an index for $(j, 2)$ games. Then $\phi$ satisfies Axiom 1, Axiom 2, Axiom 10, Axiom 11 and Axiom 12, if and only if $\phi=S$.

Proof. Existence

We know that $S$ satisfies Axiom 1, Axiom 2, Axiom $10^{8}$ and Axiom 11. In addition $S$ satisfies the shift minimal monotonicity axiom because if $p$ is a player such that $\mathcal{S M}_{p}(V) \subseteq \mathcal{S M}_{p}(W)$, then $\operatorname{sm}_{p}(V) \leq s m_{p}(W)$ which means that $S_{p}(V) s(V) \leq$ $S_{p}(W) S(W)$.

\section{Uniqueness}

Conversely, let $\phi$ be an index which satisfies Axiom 1, Axiom 2, Axiom 10, Axiom 11 and Axiom 12. Let $V$ be a $(j, 2)$ game and $p \in N$. Again, we will proceed by induction on the number of shift minimal winning $j$-partitions of $V$.

${ }^{8}$ The shift index is shift minimal additive with $\hat{I}_{p}(\mathcal{A})=\left\{\begin{array}{l}\frac{1}{s(v)} \text { if } p \notin A_{j} \\ 0 \text { otherwise }\end{array}\right.$. 
If $\mathcal{S} \mathcal{M}(V)=\left\{\left(\mathcal{A}_{1}, \ldots, \mathcal{A}_{j}\right)\right\} \mid$, that is, $|\mathcal{S} \mathcal{M}(V)|=1$, then we also have $\mathcal{S} \mathcal{M}(V)=\mathcal{M}(V)$. From Axiom 1, Axiom 2 and Axiom 10 and Axiom 11, it follows that for all $p, q \in$ $N \backslash A_{j}, \phi_{p}(V)=\phi_{q}(V)$, and for all $p \in A_{j}, \phi_{p}(V)=0$; therefore we obtain:

$$
\phi_{p}(V)=\left\{\begin{array}{l}
\frac{1}{\left|N \backslash A_{j}\right|} \text { if } p \in N \backslash A_{j} \\
0 \text { otherwise }
\end{array}\right.
$$

Note that $N \backslash A_{j} \neq \emptyset$ because from the definition of a $(j, 2)$ game, $(\emptyset, \ldots, \emptyset, N) \notin$ $\mathcal{W}(V)$.

On the other hand, we know that $S_{p}(V)=\left\{\begin{array}{l}\frac{1}{\left|N \backslash S_{j}\right|} \text { if } p \in N \backslash A_{j} \\ 0 \text { otherwise }\end{array}\right.$.

Thus, $\phi_{p}(V)=S_{p}(V)$.

Now assume that $\phi_{p}(V)=S_{p}(V)$ holds for all games $V$ for which there are at most $r-1$ shift minimal winning $j$-partitions and consider a game $V$ with $r>1$ shift minimal winning $j$-partitions, say $\mathcal{A}^{1}, \mathcal{A}^{2}, \ldots, \mathcal{A}^{r}$.

Now, set $T=\bigcap_{t=1}^{r}\left(N \backslash A_{j}^{t}\right)$ and let $p \in N$ :

- If $p \notin T$.

Consider any $(j, 2)$ game $V_{0}$ such that $\mathcal{S} \mathcal{M}\left(V_{0}\right)=\left\{\mathcal{A}^{t}: p \in N \backslash A_{j}^{t}\right\}$.

By definition of $V_{0}$, it follows that

$$
\begin{aligned}
\mathcal{S M}_{p}(V)=\left\{\mathcal{A} \in \mathcal{S} \mathcal{M}(V): p \notin A_{j}\right\} & =\left\{\mathcal{A} \in \mathcal{S M}(V): p \in N \backslash A_{j}\right\} \\
& =\left\{\mathcal{A}^{t}: p \in N \backslash A_{j}^{t}\right\} \\
& =\left\{\mathcal{A} \in \mathcal{S} \mathcal{M}\left(V_{0}\right): p \notin A_{j}\right\} \\
& =\mathcal{S M}_{p}\left(V_{0}\right)
\end{aligned}
$$

which, by Axiom 12 yields $\phi_{p}\left(V_{0}\right) s\left(V_{0}\right)=\phi_{p}(V) s(V)$.

Since $p \notin T$, the $(j, 2)$ game $V_{0}$ has less than $r$ shift minimal winning $j$-partitions. Thus, $\phi_{p}\left(V_{0}\right)=S_{p}\left(V_{0}\right)$ owing to the induction hypothesis. On the other hand, as $S$ satisfies Axiom 12, we have $S_{p}\left(V_{0}\right) s\left(V_{0}\right)=S_{p}(V) s(V)$.

We conclude that $S_{p}(V) s(V)=S_{p}\left(V_{0}\right) s\left(V_{0}\right)=\phi_{p}\left(V_{0}\right) s\left(V_{0}\right)=\phi_{p}(V) s(V)$.

This implies that $S_{p}(V)=\phi_{p}(V)$.

-If : $p \in T:$

By Axiom 11, we have $\phi_{p}(V)=\phi_{q}(V)$ and $S_{p}(V)=S_{q}(V)$ for all $p, q \in T$.

Furthermore, for all $u \notin T, \phi_{u}(V)=S_{u}(V)$. By efficiency (of $\phi$ and $S$ ), we have :

$\sum_{u \in T} \phi_{u}(V)+\sum_{u \notin T} \phi_{u}(V)=1=\sum_{u \in T} S_{u}(V)+\sum_{u \notin T} S_{u}(V)$.

This implies that $\sum_{u \in T} \phi_{u}(V)=\sum_{u \in T} S_{u}(V)$, that is, $|T| \phi_{u}(V)=|T| S_{u}(V)$ and finally, $\phi_{u}(V)=S_{u}(V)$. 


\section{Concluding discussion}

\section{On the characterization}

The null player, efficiency, additivity and homogeneity axioms appear in all characterizations presented above.The homogenity and the additivity axioms depend on the set of minimal winning $j$-partition considered. In addition to these four properties, which use only one game in their definitions, another property is necessary to characterize each of the indices presented in this paper. This last property must be one of the group of properties that establish an inequality between two games (monotonicity properties) or an equality between the union game and the component games (mergeability properties). Depending on the property used, each one of the three indices is characterized.

\begin{tabular}{|c|c|c|}
\hline $\mathcal{S}$ & $\mathcal{H}$ & $\mathcal{N P \mathcal { F }}$ \\
\hline \hline Shift monotonicity & PGI monotonicity or Mergeability & NPF monotonicity \\
Shift minimal Additivity & Minimal Additivity & NPF minimal Additivity \\
Shift minimal Homogeneity & Minimal Homogeneity & NPF minimal Homogeneity \\
Efficiency & Efficiency & Efficiency \\
Null player & Null player & Null player \\
\hline
\end{tabular}

\section{Example}

To illustrate the different indices, we will study the following $(3,2)$ game with $N=$ $\{1,2,3,4\}$ and the set of minimal winning $j$-partitions is given by

$\mathcal{M}(V)=\{(13,2,45),(5,12,34),(12,3,45)\}$. Note that, in this game, 4 is a null player and we have the following desirability relations: $1>2>3>4$ and $5>4$. The different kinds of winning coalitions are depicted in the following figure: 


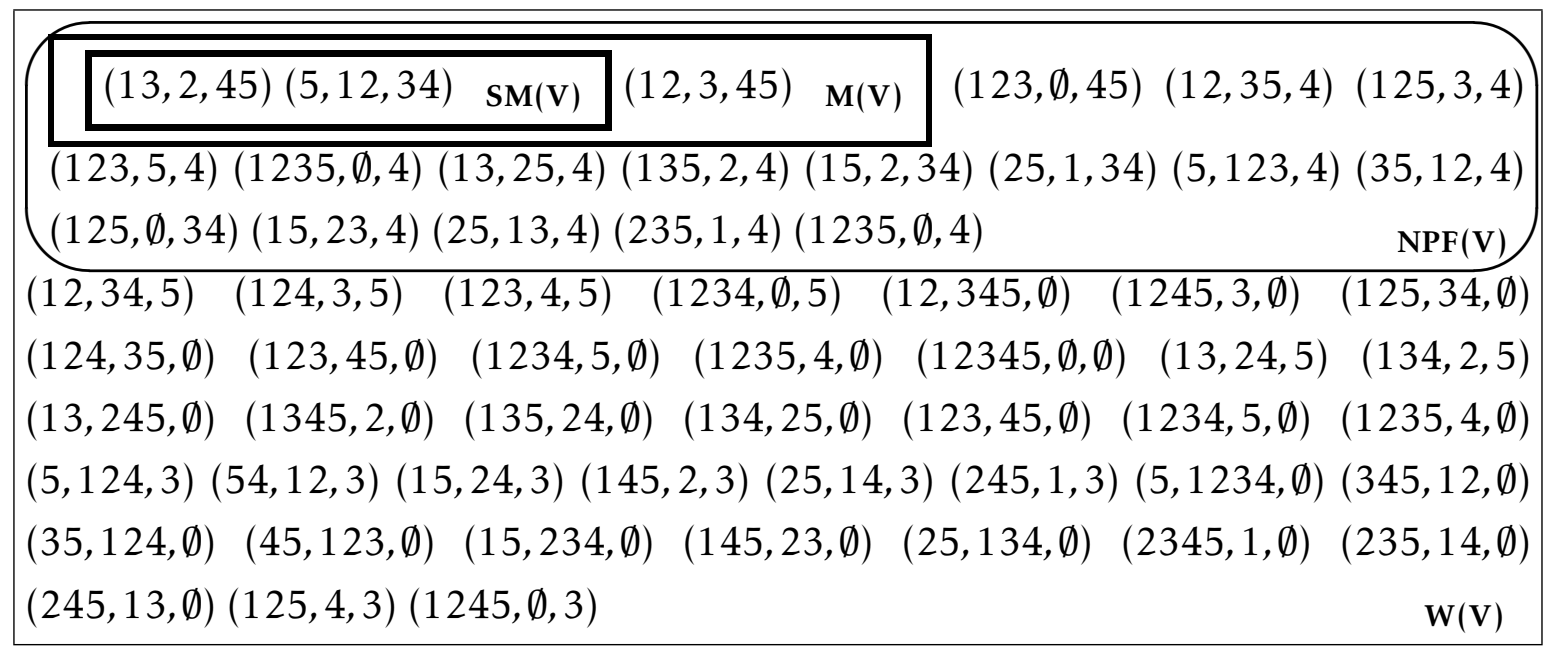

After taking into account the different sets of minimal winning $j$-partitions of each one of the players, we obtain the following indices:

$$
\begin{aligned}
H(V) & =\left(\frac{3}{9}, \frac{3}{9}, \frac{2}{9}, 0, \frac{1}{9}\right) \\
S(V) & =\left(\frac{2}{6}, \frac{2}{6}, \frac{1}{6}, 0, \frac{1}{6}\right) \\
N P F(V) & =\left(\frac{19}{70}, \frac{19}{70}, \frac{16}{70}, 0, \frac{16}{70}\right)
\end{aligned}
$$

We observe that the $j$-partition $(12,3,45)$ is not a stable partition and should not be taken into account to evaluate power with the Shift index. In this $j$-partition the level of approval of player 2 is greater than that of player 3, and player 3 is weaker than player 2 according to the desirability relation. Player 1 prefers to be with a weaker player (player 3 ) in $A_{1}$, since the partition $(13,2,45)$ is still winning. Therefore, player 3 looses power. We can remark that the weakest players (player 3 and 4) have more power with the Null player free index than with the Public good index, as it was already the case with simple game.

\section{Alternative PGI generalization}

Our PGI generalization to $(j, 2)$ games seems to us to respect the spirit of the original $P G I$ index for simple game. In our approach, players who dot not belong to the last level of approval in a minimal winning $j$-partition receive the same amount of power. An alternative generalization consists to make distinction between the different level of approval.

For all $(j, 2)$ game $V$, for all $l \in\{1,2, \ldots, j\}$, denote by $c_{p}^{*}(V)=\sum_{A \in \mathcal{M}(V)} \frac{2(j-\mathcal{A}(p))}{j(j-1)}$ and $c^{*}(V)=\sum_{p \in N} c_{p}^{*}(V)$. 
The rational number $\frac{2(j-\mathcal{A}(p))}{j(j-1)}$ can be seen as the fraction of power a player $p$ receives when she chooses a level of approval $l$ in a minimal winning $j$-partiton $\mathcal{A}$. Note that this fraction is equal to zero when a player chooses the last level of support $j$, which is consistant with Holler [1982]'s approach. For example, for $\mathcal{A}=$ $\left(A_{1}, A_{2}, A_{3}\right)$, a player who choose $A_{1}$ will be rewarded with $\frac{2}{3}$ of the power, a player who chooses $A_{2}$ obtains $\frac{1}{3}$, whereas a player in $A_{3}$ has no fraction of power.

An alternative definition of the PGI for $(j, 2)$ games is given by

$$
H_{p}^{*}(V)=\frac{c_{p}^{*}(V)}{c^{*}(V)} .
$$

According to these new index, players belonging to the same level of contribution get the same fraction of power; and more the contribution is high, more the fraction of power is high.

We can now characterize this new index. It is easy to show that the null player and the efficiency axioms are satisfied by this alternative index. Concerning the merger axiom, only the weighted are changed:

Axiom 13. (Mergeability*)

$V$ and $W$ mergeable implies that for all $p \in N$,

$$
\phi_{p}(V \vee W)=\frac{c^{*}(V)}{c^{*}(V \vee W)} \phi_{p}(V)+\frac{c^{*}(W)}{c^{*}(V \vee W)} \phi_{p}(W)
$$

And finally we introduce a new axiom which is formalised below:

Axiom 14. (Proportionnality ${ }^{*}$ )

$\phi$ satisfies Proportionality for $(j, 2)$ - games if for any $\mathcal{A} \in M(V)$ and for any $p, q \in N \backslash A_{j}$,

$$
\frac{I_{p}(\mathcal{A})}{j-\mathcal{A}(p)}=\frac{I_{q}(\mathcal{A})}{j-\mathcal{A}(q)}
$$

According to this axiom, in all minimal winning configuration in which players does not choose the last level of approval, their power are proportionnal to the level of approval they choose. Note that, when there is only one minimal winning configuration, this axiom implies that the power of two individuals who choose the same level of approval is the same. Moreover if two individuals choose always the same level of approval, their power is equivalent. We obtain the following result:

Theorem 5. Let $\phi$ be an index for $(j, 2)$ games. Then $\phi$ satisfies Axiom 1, Axiom 2, Axiom 3, Axiom 13 and Axiom 14, if and only if $\phi=H^{*}$. 


\section{Proof. Existence}

$H^{*}$ satisfies Null player : If $p$ is a null player then for all $A \in M(V), p \in A_{j}, c_{p}^{*}(V)=0$ and thus $H_{p}^{*}(V)=0$

$H^{*}$ satisfies efficiency: obvious, $\sum_{p \in N} H_{p}^{*}(V)=1$.

$H^{*}$ satisfies mergeability ${ }^{*}$ : If $V_{1}$ and $V_{2}$ are mergeable then, it is easy to verify that $c_{p}^{*}\left(V_{1} \vee V_{2}\right)=c_{p}^{*}\left(V_{1}\right) \cup c_{p}^{*}\left(V_{2}\right)$ and $c_{p}^{*}\left(V_{1}\right) \cap c_{p}^{*}\left(V_{2}\right)=\emptyset$, which implies that $c^{*}\left(V_{1} \vee V_{2}\right)=$ $c_{p}^{*}\left(V_{1}\right)+c_{p}^{*}\left(V_{2}\right)$. Therefore,

$$
\begin{aligned}
H_{p}^{*}\left(V_{1} \vee V_{2}\right)=\frac{c_{p}^{*}\left(V_{1} \vee V_{2}\right)}{c^{*}\left(V_{1} \vee V_{2}\right)} & =\frac{c_{p}^{*}\left(V_{1}\right)+c_{p}^{*}\left(V_{2}\right)}{c^{*}\left(V_{1} \vee V_{2}\right)} \\
& =\frac{c_{p}^{*}\left(V_{1}\right)}{c^{*}\left(V_{1} \vee V_{2}\right)}+\frac{c_{p}^{*}\left(V_{2}\right)}{c^{*}\left(V_{1} \vee V_{2}\right)} \\
& =\frac{H_{p}^{*}\left(V_{1}\right) \cdot c^{*}\left(V_{1}\right)}{c^{*}\left(V_{1} \vee V_{2}\right)}+\frac{H_{p}^{*}\left(V_{2}\right) \cdot c^{*}\left(V_{2}\right)}{c^{*}\left(V_{1} \vee V_{2}\right)} \\
& =\frac{c^{*}\left(V_{1}\right)}{c^{*}\left(V_{1} \vee V_{2}\right)} H_{p}^{*}\left(V_{1}\right)+\frac{c^{*}\left(V_{2}\right)}{c^{*}\left(V_{1} \vee V_{2}\right)} H_{p}^{*}\left(V_{2}\right)
\end{aligned}
$$

$H^{*}$ satisfies minimal additivity : By definition, for all game $V$, for all $p$, we have $H_{p}^{*}(V)=\frac{c_{p}^{*}(V)}{c^{*}(V)}$ where $c_{p}^{*}(V)=\sum_{A \in \mathcal{M}(V)} \frac{2(j-A(p))}{j(j-1)}$. Then $H_{p}^{*}(V)=\sum_{A \in \mathcal{M}(V)} \frac{\frac{2(j-A(p))}{j(j-1)}}{c^{*}(V)}=\sum_{A \in \mathcal{M}(V)} I_{p}(A)$ with $I_{p}(A)=\frac{\frac{2(j-A(p))}{j(j-1)}}{c^{*}(V)}$ and $\mathcal{F}(V)=\mathcal{M}(V)$.

$H^{*}$ satisfies proportionality : Let $V$ be a $(\mathrm{j}, 2)$ game, $A \in \mathcal{M}(V), p$ and $q$ two players such that $p, q \notin A_{j}$. We have : $\frac{I_{p}(\mathcal{A})}{j-\mathcal{A}(p)}=\frac{\frac{2(j-\mathcal{A}(p))}{j(j-1)}}{c^{*}(V)} \frac{1}{j-A(p)}=\frac{2(j-\mathcal{A}(p))}{j(j-1) \cdot c^{*}(V)} \frac{1}{j-\mathcal{A}(p)}=\frac{2}{j(j-1) \cdot c^{*}(V)}$ and $\frac{I_{q}(\mathcal{A})}{j-A(q)}=\frac{\frac{2(j-\mathcal{A}(q))}{j(j-1)}}{c^{*}(V)} \frac{1}{j-\mathcal{A}(q)}=\frac{2(j-\mathcal{A}(q))}{j(j-1) \cdot \mathcal{C}^{*}(V)} \frac{1}{j-A(q)}=\frac{2}{j(j-1) \cdot \mathcal{C}^{*}(V)}$, which implies that $\frac{I_{p}(\mathcal{A})}{j-\mathcal{A}(p)}=\frac{I_{q}(\mathcal{A})}{j-\mathcal{A}(q)}$.

\section{Uniqueness}

Conversely, let $\phi$ be an index which satisfies efficiency, null player, minimal additivity, proportionality and merger* Let $V$ be a $(j, 2)$ game and $p \in N$. As previously, we proceed by induction on the number of minimal winning $j$-partitions of $V$. Firstly, we prove that $\phi_{p}(V)=H_{p}^{*}(V)$ when $M(V)=\left\{\mathcal{A}=\left(A_{1}, A_{2}, \ldots, A_{j}\right)\right\}$ that is, $|M(V)|=1$. Since $|M(V)|=1$, then by minimal additivity $\phi_{p}(V)=I_{p}(\mathcal{A})$. From proportionnality we have $\frac{I_{p}(\mathcal{A})}{j-A(p)}=\frac{I_{q}(\mathcal{A})}{j-A(q)}$ for all $p, q \notin A_{j}$, which implies that for all $q \notin A_{j}, I_{q}(\mathcal{A})=\frac{j-A(q)}{j-A(p)} \cdot I_{p}(\mathcal{A})$. From efficiency, it follows that $\sum_{q \in N} I_{q}(\mathcal{A})=1$. Moreover $\sum_{q \in N} I_{q}(\mathcal{A})=\sum_{q \in A_{j}} I_{q}(\mathcal{A})+\sum_{q \notin A_{j}} I_{q}(\mathcal{A})=\sum_{q \notin A_{j}} I_{q}(\mathcal{A})$ because from the Null player axiom, $I_{q}(\mathcal{A})=\phi_{q}(V)=0$ for all $q \in A_{j}$. But, for all $q \notin A_{j}, \sum_{q \notin A_{j}} I_{q}(\mathcal{A})=\sum_{q \notin A_{j}} \frac{j-\mathcal{A}(q)}{j-\mathcal{A}(p)} I_{p}(\mathcal{A})=$ 
$\frac{I_{p}(\mathcal{A})}{j-\mathcal{A}(p)} \sum_{q \notin A_{j}}(j-\mathcal{A}(q)) ;$ thus, $I_{p}(\mathcal{A})=\frac{j-\mathcal{A}(p)}{\sum_{q \notin A_{j}}(j-\mathcal{A}(q))}=\phi_{p}(V)$

On the other hand, $H_{p}^{*}(V)=\frac{c_{p}^{*}(V)}{c^{*}(V)}=\frac{\frac{2(j-\mathcal{A}(p))}{j(j-1)}}{\sum_{q \in N} \frac{2(j-\mathcal{A}(q))}{j(j-1)}}=\frac{2(j-\mathcal{A}(p))}{j(j-1)} \frac{j(j-1)}{2 \sum_{q \in N}(j-\mathcal{A}(q))}=\frac{j-\mathcal{A}(p)}{\sum_{q \in N}(j-\mathcal{A}(q))}=$ $\frac{j-\mathcal{A}(p)}{\sum_{q \notin A_{j}}(j-\mathcal{A}(q))}=\phi_{p}(V)$. Thus, $H_{p}^{*}(V)=\phi_{p}(V)$.

Let $r>1$ and assume that $H_{p}^{*}(V)=\phi_{p}(V)$ holds for all game $V$ for which there are at most $r-1$ minimal winning $j$-partitions. Consider a game $V$ with $r$ minimal winning $j$-partitions, say $\mathcal{A}^{1}, \mathcal{A}^{2}, \ldots, \mathcal{A}^{r}$. The games $V_{1} \vee \ldots \vee V_{r-1}$ and $V_{r}$ are mergeable, where $V_{r}$ is the $(j, 2)$ game with the single minimal $j$-partition $\mathcal{A}^{r}$. Furthermore, $V=\left[V_{1} \vee \ldots \vee V_{r-1}\right] \vee V_{r}$ and it follows, from the merger ${ }^{*}$ axiom that

$$
\begin{aligned}
\phi_{p}(V)= & \frac{c^{*}\left(V_{1} \vee \ldots \vee V_{r-1}\right)}{c^{*}(V)} \phi_{p}\left(V_{1} \vee \ldots \vee V_{r-1}\right)+\frac{c^{*}\left(V_{r}\right)}{c^{*}(V)} \phi_{p}\left(V_{r}\right) \\
= & \frac{c^{*}\left(V_{1} \vee \ldots \vee V_{r-1}\right)}{c^{*}(V)} H_{p}^{*}\left(V_{1} \vee \ldots \vee V_{r-1}\right)+\frac{c^{*}\left(V_{r}\right)}{c^{*}(V)} H_{p}^{*}\left(V_{r}\right) \\
& (\text { thanks to the induction hypothesis) } \\
= & H_{p}^{*}\left(\left[V_{1} \vee \ldots \vee V_{r-1}\right] \vee V_{r}\right) \text { (because } H^{*} \text { satisfies the merger axiom) } \\
= & \left.H_{p}^{*}(V) \text { (because of the mergeability of } V_{1} \vee \ldots \vee V_{r-1} \text { and } V_{r}\right)
\end{aligned}
$$

In conclusion, for all game $V$ on $N$, all player $p \in N, \phi_{p}(V)=H_{p}^{*}(V)$.

\section{Extension}

There are several ways in which the public good indices could be explored in further research. First of all, we can study the extension of public good indices to other games with multiple alternatives of support. Secondly, following the works of Tchantcho et al. [2008], we can also qualitatively compare the a priori influence of voters with the three public good indices. Another interesting direction is the extension of public good indices to $(j, 2)$ games which take into account the different a priori relationships between players in the spirit of Alonso-Meijide et al. [2010] and Courtin et al. [2017].

\section{References}

J.M. Alonso-Meijide and J. Freixas. A new power index based on minimal winning coalitions without any surplus. Decision support system, 49:70-76, 2010. 
J.M. Alonso-Meijide, B. Casas-Méndez, M.J. Holler, and S. Lorenzo-Freire. Computing power indices: Multilinear extensions and new characterizations. European Journal of Operational Research, 188:540-554, 2008.

J.M. Alonso-Meijide, B. Casas-Méndez, G. Fiestras-Janeiro, M.J. Holler, and A. Nohn. Axiomatizations of public good indices with a priori unions. Social Choice and Welfare, 35:517-533, 2010.

J.M. Alonso-Meijide, M. Álvarez-Mozos, F. Ferreira, and A. Pinto. Two new power indices based on winning coalitions. Journal of Difference Equations and Applications, 17:1095-1100, 2011.

M. Álvarez-Mozos. Essays on cooperative games with restricted cooperative and simple games. PhD Thesis, University of Santiago de Compostela, Spain, 2012.

N. Andjiga, F. Chantreuil, and D. Lepelley. La mesure du pouvoir de vote. Mathematiques et Sciences Humaines, 163:111-145, 2003.

J.F. Banzhaf. Weighted voting doesn't work: A mathematical analysis. Rutgers Law Review, 19:317-343, 1965.

B. Barry. Is it better to be powerful or lucky: part i and part ii. Political Studies, 28: 183-194 and 338-352, 1980.

S. Courtin, Z. Nganmeni, and B. Tchantcho. The Shapley-Shubik power index for dichotomous multi-type games:. Theory and Decision, 81:413-426, 2016.

S. Courtin, Z. Nganmeni, and B. Tchantcho. Dichotomous multi-type games with a coalition structure. Mathematical Social Sciences, 86:9-17, 2017.

J. Deegan and E.W. Packel. A new index of power for simple n-person games. International Journal of Game Theory, 7:113-123, 1978.

J. Freixas. The Banzhaf index for games with several levels of approval in the input and output. Annals of Operations Research, 137:45-66, 2005a.

J. Freixas. The Shapley Shubik power index for games with several levels of approval in the input and output. Decision Support Systems, 39:185-195, 2005b.

J. Freixas and W. S. Zwicker. Weighted voting, abstention, and multiple levels of approval. Social Choice and Welfare, 21:399-431, 2003a. 
J. Freixas and W.S. Zwicker. Weighted voting, abstention, and multiple levels of approval. Social Choice and Welfare, 21:399-431, 2003b.

M.J. Holler. Forming coalitions and measuring voting power. Political Studies, 30: 262-271, 1982.

M.J. Holler and E.W. Packel. Power, luck and the right index. Journal of Economics, 43:21-29, 1983.

J.R. Isbell. A class of simple games. Duke Mathematical Journal, 25:423-439, 1958.

A. Laruelle and F. Valenciano. Voting and collective decision-making. Cambridge University Press, 1st edition, 2008.

R. Pongou, B. Tchantcho, and L. Diffo Lambo. Political influence in multi-choice institutions: cyclicity, anonymity, and transitivity. Theory and Decision, 70:157178, 2011.

R. Pongou, B. Tchantcho, and L. Tedjeugang. Power theories for multi-choice organizations and political rules: Rank-order equivalence. Operations Research Perspectives, 1:42-49, 2014.

R. Pongou, B. Tchantcho, J.S Zwicker, and N. Tedjeugang. Properties of ladder tournaments. European Journal of Operational Research, 263:203-213, 2017.

W.H. Riker. Theory of political coalitions. Journal Studies, 30:262-271, 1982.

L. S. Shapley and M. Shubik. A model for evaluating the distribution of power in a committee system. Rutgers Law Review, 48:787-792, 1954.

B. Tchantcho, L. Diffo Lambo, and R. Pongou. Voters' power in voting games with abstention: Influence relation and ordinal equivalence of power theories. Games and Economic Behavior, 64:335-350, 2008. 